\title{
Clinical significance of Iroquois Homeobox Gene - IRX1 in human glioma
}

\author{
PENGXING ZHANG ${ }^{1 *}$, NAN LIU ${ }^{1 *}$, XIAOSHAN XU ${ }^{1}$, ZHEN WANG $^{1}$, YINGDUAN CHENG ${ }^{1,2}$, \\ WEILIN JIN ${ }^{3}$, XIN WANG ${ }^{4}$, HONGWEI YANG ${ }^{4}$, HUI LIU ${ }^{1}$, YONGSHENG ZHANG ${ }^{1}$ and YANYANG TU ${ }^{1}$ \\ ${ }^{1}$ Department of Experimental Surgery, Tangdu Hospital, Fourth Military Medical University, Xi'an, Shaanxi 710038, \\ P.R. China; ${ }^{2}$ Department of Research, Cipher Ground, 675 Rt 1 South, North Brunswick, NJ 08902, USA; ${ }^{3}$ Institute \\ of Nano Biomedicine and Engineering, Department of Instrument Science and Engineering, Key Laboratory \\ for Thin Film and Microfabrication Technology of Ministry of Education, School of Electronic Information \\ and Electronic Engineering, Shanghai Jiao Tong University, Shanghai 200240, P.R China; ${ }^{4}$ Department of \\ Neurosurgery, Brigham and Women's Hospital, Harvard Medical School, Boston, MA, 02115, USA
}

Received April 7, 2017; Accepted January 2, 2018

DOI: $10.3892 / \mathrm{mmr} .2018 .8404$

\begin{abstract}
The present study aimed to investigate the location, expression and clinical significance of Iroquois homeobox gene (IRX1) in human glioma. The expression of IRX1 gene in glioma cell lines (U87, U373, LN229 and T98G) and normal brain tissue was detected via reverse transcription-polymerase chain reaction. The IRX1 protein in fresh glioma specimens, with the adjacent normal brain tissue, was quantified through western blotting. The archived glioma only specimens from the present hospital and glioma specimens with adjacent normal brain tissue, from Alenabio biotechnology, were subjected to immunohistochemistry and tissue microarray analysis, respectively. The Kaplan-Meier method was employed to assess the correlation between the IRX1 level and the overall survival time of the patients. IRX1 gene was demonstrated to be expressed at varying levels in U373, LN229 and T98G cells, however not in U87 cells and normal brain tissue. Western blotting revealed increased IRX1 expression in glioma tissue compared with adjacent normal brain tissue. Furthermore, a direct correlation was observed between the IRX1 expression and the clinical glioma grade, with a significant difference in the gene expression between high grade and low grade glioma $(\mathrm{P}<0.05)$. Notably, IRX1 was identified to be localized to the cytoplasm in the adjacent normal brain and World Health Organization grade I glioma, whereas was identified to be present in the nucleus in higher grade glioma. In addition to being established as a significant prognostic vari-
\end{abstract}

Correspondence to: Professor Yongsheng Zhang or Professor Yanyang Tu, Department of Experimental Surgery, Tangdu Hospital, Fourth Military Medical University, 1 Xinsi Road, Xi'an, Shaanxi 710038, P.R. China

E-mail: Zhangys_td@163.com

E-mail: tu.fmmu@gmail.com

Key words: IRX1 gene, Human glioma, Gene expression, survival able, IRX1 expression was positively correlated with the overall survival of glioma patients. IRX1 gene may therefore exhibit an oncogenic role in glioma condition, and thus may be of clinical importance as a future therapeutic target.

\section{Introduction}

Malignant glioma, a malignant neoplasm of the central nervous system, is a devastating condition associated with poor prognosis. Owing to the location and aggressive nature of glioma, it is extremely difficult to achieve the complete surgical resection of the tumor, inevitably leading to the recurrence (1). Statistics revealed that the five-year survival rate of glioma patients is less than $30 \%$, with the survival rate of WHO grade IV glioma patients being as low as six months (2). Considering the aggressiveness and devastating nature of the disease, it is critical to develop an effective treatment. In this regards, identifying the genes that are closely related to the genesis, development and prognosis of glioma is crucial.

IRX1 gene is a member of Iroquois homeobox gene family, closely associated with the embryonic development in both vertebrates and invertebrates. IRX1 protein, encoded by IRX1 gene, acts as transcription factor, which in combination with other family proteins, actively participates in nervous system development (3). A recent study identified the expression of IRX1 gene during early lung development in rats, the overexpression of which was correlated to pulmonary hypoplasia (4). IRX1 also plays a critical role in cancer condition, which may vary from being tumor suppressor gene, prometastatic gene or tumor oncogene under different types of cancer (5). However, the role of IRX1 gene in human glioma is not clear yet, and the same is being addressed in the present study, by exploring its expression and clinical significance in glioma.

\section{Materials and methods}

This study was approved by the Research Ethics Committee of Tangdu Hospital, Fourth Military Medical University, 
P.R. China. Written informed consent was obtained from the participating patients. All clinical data/specimens were anonymized and handled as per the related ethical and legal standards.

Cells and tissue samples. Human glioma cells (U87, U373, LN229 and T98G) were cultured in Dulbecco's modified eagle's medium (DMEM), supplemented with $10 \%$ fetal calf serum (GIBCO, USA) and $1 \%$ Penicillin/Streptomycin (SIGMA ALDRICH, USA), and incubated at $37^{\circ} \mathrm{C}$ with $5 \%$ $\mathrm{CO}_{2}$. It should be specially noted that the origin of the U87 cell line is unknown, but it is a likely glioblastoma cell line (6). And U373 cells are known to be a U251 derivative (7), obtained from ATCC (HTB-17).

All the study specific tissue samples were collected from the glioma patients treated at the department of neurosurgery, Tangdu hospital, Fourth Military Medical University, P.R. China. These tissue samples comprised of 4 glioma (1G: WHO IV, 2G: WHO III, 3G: WHO III-IV, 4G: WHO IV) +4 adjacent normal brain tissue specimens, freshly collected from the surgeries during 2015 and 54 glioma only specimens, archived under liquid nitrogen at the pathology department, from the surgeries during $01 / 2008$ to $12 / 2010$. None of the patients had received chemotherapy or radiotherapy before surgery. The fresh specimens were snap-frozen in liquid nitrogen and stored at $-80^{\circ} \mathrm{C}$, while archived glioma only specimens were fixed in $10 \%$ buffered formalin and embedded in paraffin, till further analysis.

Total RNA isolation and cDNA synthesis. The total RNA from human glioma cell lines (U87, U373, LN229 and T98G) was purified using TRIzol reagent. Normal brain tissue RNA was purchased from Clontech. Typically, $1 \mu \mathrm{g}$ of the total RNA was used to generate cDNA by employing SuperScript ${ }^{\circledR}$ II RT (TAKARA,JAPAN) with oligo-dT primer. The PCR primers for IRX1 and GAPDH were 5'-TCATTGACCTCAACTACATG-3' (F) and 5'-TCGCTCCTGGAAGATGGTGAT-3' (R), and 5'-CTCAGCCTCTTCTCGCAGAT-3' (F) and 5'-TCTTCCTGGTCCTTGCTGC-3' (R), respectively (8). Each PCR was performed for 30 thermal cycles and the PCR products were observed by electrophoresis on a $1.5 \%$ agarose gel, visualized after staining with ethidium bromide.

The expression of IRX1 protein in glioma, detected by Western Blot. The IRX1 protein expression in the glioma condition was assessed using the fresh glioma tissues, along with the adjacent normal brain tissue. The total protein from fresh tissues, separated into glioma tissue and adjacent normal tissue, were extracted via mammalian protein extraction reagent (Pierce, Appleton, WI, USA) supplemented with protease inhibitors cocktail (Sigma, USA). The total protein concentrations were determined via BCA protein assay kit (Boster Systems, Inc., Pleasanton, CA, USA). The proteins were separated by $10 \%$ SDS-PAGE (Genshare biological, shaanxi, China) and then transferred to PVDF membranes (Millipore, Boston, USA). The membranes were blocked with TBST buffer (TBS plus $0.1 \%$ Tween-20) containing 5\% w/v non-fat milk, and hybridized with IRX1 antibody (Code NO. Ab180860, Abcam, USA) at 1:1500 dilution, followed by incubation with specific HRP-conjugated secondary antibody (1:2500, Bioworld, St.
Table I. Characteristic analysis of patients corresponding to glioma specimen.

\begin{tabular}{lccr}
\hline & & \multicolumn{2}{c}{ IRX1 expression } \\
\cline { 3 - 4 } $\begin{array}{l}\text { Clinico-pathological } \\
\text { features }\end{array}$ & $\begin{array}{c}\text { No. of } \\
\text { cases }\end{array}$ & low (n. \%) & high (n. \%) \\
\hline WHO grade & & & \\
I & 5 & $4(80)$ & $1(20)$ \\
II & 16 & $13(82)$ & $3(18)$ \\
III & 14 & $3(21)$ & $11(79)$ \\
IV & 19 & $2(10)$ & $17(90)$ \\
Age & & & \\
$<55$ & 30 & $17(57)$ & $13(43)$ \\
$\geq 55$ & 24 & $5(21)$ & $19(79)$ \\
Gender & & & \\
Male & 33 & $13(39)$ & $20(61)$ \\
Female & 21 & $9(43)$ & $12(57)$ \\
\hline
\end{tabular}

Louis, USA). Proteins were visualized using ECL detection system (Amersham Bio-sciences, Uppsala, Sweden).

The expression and location of IRX1 protein in glioma tissues via immunohistochemistry (IHC) and tissue microarray. The 54 glioma only specimens were subject to IHC analysis, by DAB detection kit (Streptavidin-Biotin; ZSGB-BIO, Beijing, China), to assess the expression and location of IRX1. Briefly, following a peroxidase block with $3 \% \mathrm{H}_{2} \mathrm{O}_{2} /$ methanol for $30 \mathrm{~min}$, specimens were blocked with $5 \%$ normal goat serum. Then the slides were incubated overnight, at $4^{\circ} \mathrm{C}$, with mouse polyclonal antihuman IRX1 primary antibody (Code NO. BS2291, Bioworld, USA), at 1:50 dilution. Then, the specimens were briefly washed for 3 times with PBS and incubated at room temperature with the anti-goat secondary antibody for $1 \mathrm{~h}$, followed by incubation with $\mathrm{HRP} / \mathrm{Fab}$ polymer conjugate for $30 \mathrm{~min}$, at $37^{\circ} \mathrm{C}$. The signal visualization was performed by treating with $\mathrm{DAB}$ chromogen for 2 to $3 \mathrm{~min}$. After water wash, specimens were counterstained with Meyer's hematoxylin (ZSGB-BIO).

Tissue microarray assay (NO. BS17017b, alenabio) was employed to further validate the IHC results. A total of 62 brain tissue samples were included in the assessment and were classified as; 24 low-grade gliomas [3 pilocytic astrocytomas (WHO I) and 21 diffuse astrocytomas (WHO II)], 33 high-grade gliomas [9 anaplasia astrocytomas (WHO III) and 24 primary glioblastomas (WHO IV)] and 5 adjacent normal brain tissue. All the samples were provided and analyzed, through IHC, by Alenabio biotechnology LTD, Xi'an, China. The immunostaining was performed using mouse polyclonal anti-human IRX1 primary antibody (Bioworld, USA), at 1:50 dilution, while the same was replaced by non-immune IgG antibody for negative control samples. All specimens were counterstained with Meyer's hematoxylin.

The qualitative and semi-quantitative evaluation of the IHC and tissue microarray samples were achieved as follows: five random zones of the stained glioma samples were observed under the light microscope (high power field) and assessed the 


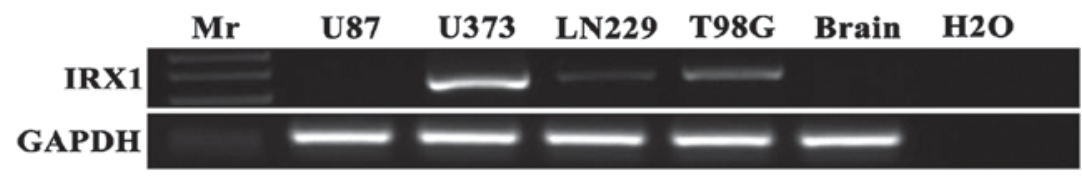

Figure 1. The expression of IRX1 in glioma cell lines and normal tissues, analyzed by PCR. Mr: 100 bp DNA Ladder Marker.

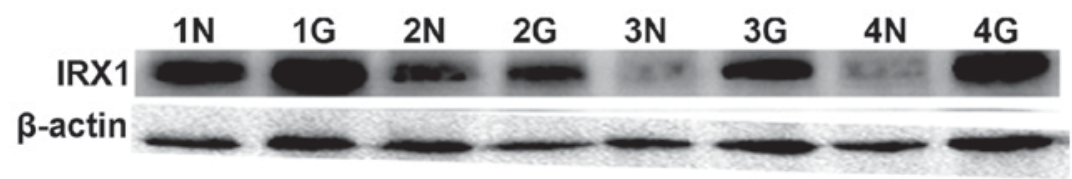

Figure 2. The protein expression level of IRX1 in glioma tissues, detected by Western blot analysis. N: Normal tissue, G: Glioma tissue. Tissues in the same group are from same patient.

location of the stain, intensity of staining and the percent of positively stained cells. Based on the intensity and the percent of positively stained cells the samples were scored as follows: no staining as negative (-), $<25 \%$ positive cells as weakly positive (+), $25 \%-49 \%$ of positive cells as moderately positive $(++)$, and $>50 \%$ of positive cells as strong positive $(+++/++++)$. The results were evaluated according to WHO classifications by two experienced ( $>10$ years) pathologists, with differences resolved through careful discussion.

Clinico-pathological features, prognostic variables and overall patient survival. The clinico-pathological features of the participating patients were collected from their respective clinical records and the histopathological classification of their glioma condition (WHO classification) was re-evaluated using the study specific H\&E staining. The data were correlated to the IRX1 expression and are listed in Table I. The pathological variables affecting the prognosis of the glioma condition were also assessed, through univariate and multivariate analysis.

Further, the survival time of all the 54 patients, from whom glioma only specimens were obtained, was assessed by retrospectively following up for a median period of 60 months. The overall survival time, defined as the time from the date of the initial surgery to death, was calculated based on the Kaplan-Meier method. Patients who died of reasons not related to glioma were excluded from the analysis.

Statistical analysis. SPSS 17.0 software (SPSS Inc., Chicago, IL, USA) was applied for all statistical analyses, and a P-value $<0.05$ was considered as significant.

\section{Results}

The expression level of IRX1 gene in glioma cells. As per the RT-PCR results, all the glioma cell lines (U373, LN229 and T98G cells), except U87 cells, expressed IRX1, while the same was not detected in normal brain tissue (Fig. 1). This suggests of a probable positive correlation of IRX1 with glioma.

The expression and location of IRXI in glioma tissues. Western blot assay showed an increased expression of IRX1 protein in glioma tissue, compared to adjacent normal brain tissue, with the difference reaching statistical significance $(\mathrm{P}<0.05)$ (Fig. 2). The data was consistent with our finding in cell lines, which further confirmed a correlation of IRX1 with glioma .

Histologically, among 54 cases of glioma, 25 cases were classified as low-grade gliomas [6 pilocytic astrocytomas (WHO I) and 19 diffuse astrocytomas (WHO II)], and 29 cases as high-grade gliomas [12 anaplasia astrocytomas (WHO III) and 17 primary glioblastomas (WHO IV)]. The IHC analysis of glioma samples revealed that the IRX1 protein was expressed in all grades of glioma tissues. Interestingly the localization of IRX1 shifted from cytoplasm in WHO grade I glioma tissue to nucleus in the higher grade gliomas (Fig. 3A). Further, a positive correlation between IRX1 expression and the glioma grade was observed.

Our IHC results were reconfirmed through tissue microarray (NO.IHC 160917), which, in addition to confirming the expression of IRX1 in glioma tissues, also revealed its presence in the adjacent normal brain tissue. A clear shift in the location of IRX1 from cytoplasm in adjacent normal brain tissue and WHO grade I glioma tissue to nucleus in the higher grade gliomas was observed (Table IIA and Fig. 4), where it took the form of rough trachychromatic claybank granules. Interestingly, one sample in the WHO grade II glioma specimen displayed co-localization of IRX1 in both cytoplasm and nucleus. A few glioma samples with no IRX1 expression was also seen, the number of which sequentially increased with the increase in glioma grade. Among the assessed glioma tissue samples, WHO grade I glioma tissues were restricted to weakly positive IRX1 expression while few WHO grade II and III samples managed to increase IRX1 expression to moderate positivity. Compared to the other glioma grades, significant number of WHO grade IV glioma tissues displayed moderately positive IRX1 expression, with one sample even managing to display strong positivity (Table IIB). The localization difference in tissue microarray analysis was more obvious than that in IHC, and the testing results of normal brain tissues were added in tissue microarray, which is a supplement and further verification of IHC. These results reconfirmed a possible correlation of IRX1 expression with glioma grades.

Prognostic variables and correlation of IRXI expression with overall survival of glioma patients. Univariate analysis showed that large tumor diameter $(\mathrm{P}=0.04)$, intra-tumor necrosis $(\mathrm{P}=0.02)$ and high IRX1 expression $(\mathrm{P}=0.008)$ were significantly associated with poor prognosis. Further multi- 
Table II. Cellular location and expression of IRX1 in brain tissue and glioma tissue.

A, The cellular location of IRX1 in glioma and adjacent normal brain tissue

\begin{tabular}{lcccc}
\hline & $\begin{array}{c}\text { Adjacent } \\
\text { glioma tissue } \\
\text { brain tissue }\end{array}$ & $\begin{array}{c}\text { Grade I } \\
\text { glioma } \\
\text { tissue }\end{array}$ & $\begin{array}{c}\text { Grade II } \\
\text { glioma } \\
\text { tissue }\end{array}$ & $\begin{array}{c}\text { Grade III } \\
\text { glioma } \\
\text { tissue }\end{array}$ \\
$\begin{array}{c}\text { Grade IV } \\
\text { glioma } \\
\text { tissue }\end{array}$ \\
Number of cells expressing IRX1 in cytoplasm & 5 & 2 & $\begin{array}{c}\text { 1(in cytoplasm } \\
\text { and nucleus) }\end{array}$ & 0 \\
Number of cells expressing IRX1 in nucleus & 0 & 0 & 18 & 6 \\
Number of cells not expressing IRX1 & 0 & 1 & 2 & 3 \\
Total number of cells assessed & 5 & 3 & 21 & 4 \\
\hline
\end{tabular}

B, The expression of IRX1 in brain tissue and glioma tissue

\begin{tabular}{lcccc}
\hline & $\begin{array}{c}\text { Negative } \\
(-)\end{array}$ & $\begin{array}{c}\text { Weak } \\
\text { positive (+) }\end{array}$ & $\begin{array}{c}\text { Moderately } \\
\text { positive (++) }\end{array}$ \\
\hline Adjacent brain tissue (cytoplasm) & 0 & 1 & 3 & 1 \\
WHO I (cytoplasm) & 1 & 2 & 0 & 0 \\
WHO II (nucleus) & 2 & 14 & 5 & 0 \\
WHO III (nucleus) & 3 & 14 & 2 & 0 \\
WHO IV (nucleus) & 4 & 10 & 9 & 1 \\
\hline
\end{tabular}

Table III. Univariate and multivariate analyses.

\begin{tabular}{|c|c|c|c|c|c|c|}
\hline \multirow[b]{2}{*}{ Parameter } & \multicolumn{3}{|c|}{ Univariate analysis } & \multicolumn{3}{|c|}{ Multivariate analysis } \\
\hline & Risk ratio & $\begin{array}{l}95 \% \text { confidence } \\
\text { interval }\end{array}$ & $\mathrm{P}$-value & Risk ratio & $\begin{array}{c}95 \% \text { confidence } \\
\text { interval }\end{array}$ & P-value \\
\hline Age & 0.90 & $0.22-1.97$ & N.S. & - & - & - \\
\hline Gender & 1.11 & $0.56-2.69$ & N.S. & - & - & - \\
\hline Largest tumor diameter & 2.08 & $1.03-4.62$ & 0.04 & 1.12 & $0.58-2.88$ & N.S. \\
\hline Intra-tumor necrosis & 2.82 & $1.02-6.09$ & 0.02 & 2.03 & $1.02-4.92$ & 0.04 \\
\hline IRX1 expression & 4.60 & $1.12-10.29$ & 0.008 & 3.20 & $1.06-6.98$ & 0.01 \\
\hline
\end{tabular}

variate analysis, based on Cox proportional hazards model, identified intra-tumor necrosis $(\mathrm{P}=0.04)$ and IRX1 expression $(\mathrm{P}=0.01)$ as two independent prognostic variables. Statistical values of the expression of IRX1 and other clinical parameters derived from Cox stepwise proportional hazards model are indicated in Table III.

In total, all the 54 patients had a complete follow-up record. The Kaplan-Meier analysis demonstrated a significant association of IRX1 expression with the overall survival of glioma patients (Fig. 3B).

\section{Discussion}

In the present study, we assessed the clinical significance of IRX1 in glioma condition. First, through RT-PCR, we found that most of the assessed human glioma cell lines (U373, LN229 and T98G) expressed IRX1, while the same was not detected in normal brain tissue (Fig. 1). Western Blot analysis showed an increased expression of IRX1 protein in glioma tissue that was significantly higher than the adjacent brain tissue (Fig. 2). Further, the IHC and tissue microarray demonstrated a positive correlation of the IRX1 expression with the glioma grade. Further, the localization of IRX1 was found shifted from the cytoplasm in the WHO I glioma and adjacent normal brain tissue, to nucleus in the higher grade gliomas (Figs. 3A and 4). Further to being established as a prognostic variable, the IRX1 expression was positively correlated with the overall survival of glioma patients (Fig. 3B). The above results indicate that IRX1 might play an important role in the development of glioma, and be of potential use in assessing the prognosis and malignancy status of the condition and also as a target for molecular therapy of glioma. In this study, The normal brain tissue RNA used for RT-PCR was purchased from Clontech. And there is no protein in normal brain tissue 
$\mathbf{A}$

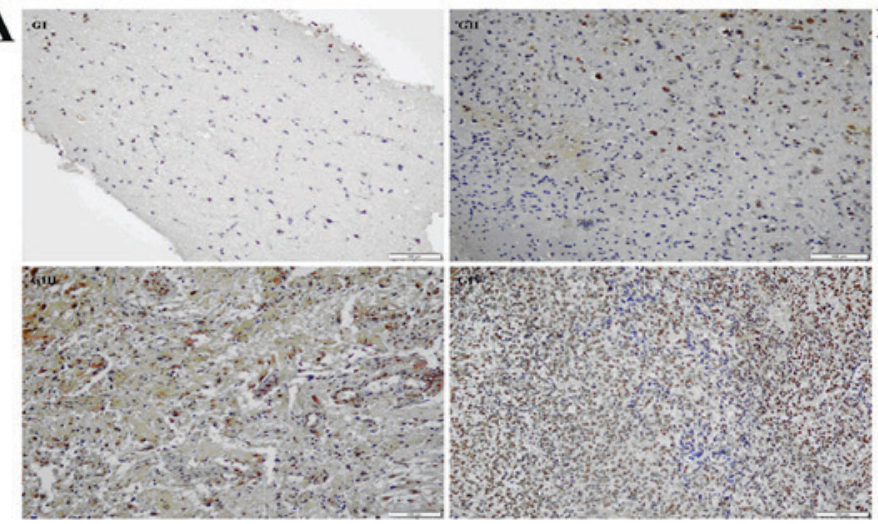

B

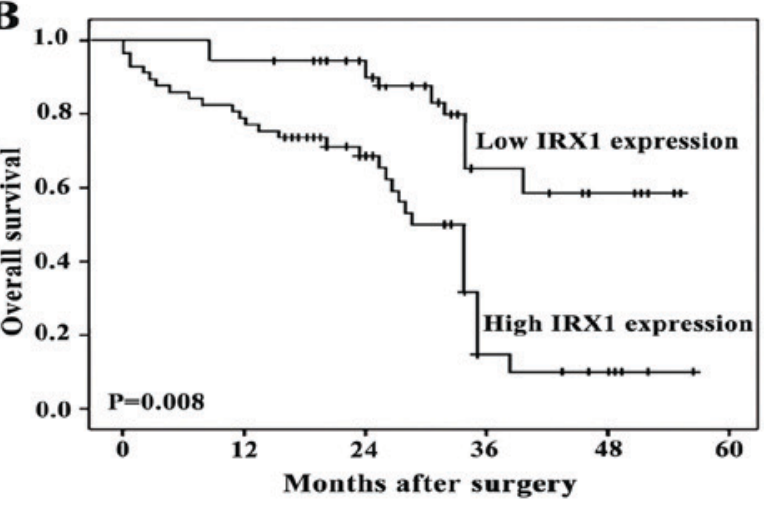

Figure 3. (A) The protein expression level of IRX1 in glioma tissues, detected by IHC analysis. GI: Glioma WHO I (+), GII: Glioma WHO II (++), GIII: Glioma WHO III (+++), GIII: Glioma WHO IV (+++). (B) Cumulative survival curves of the patients according to the expression of IRX1 protein.

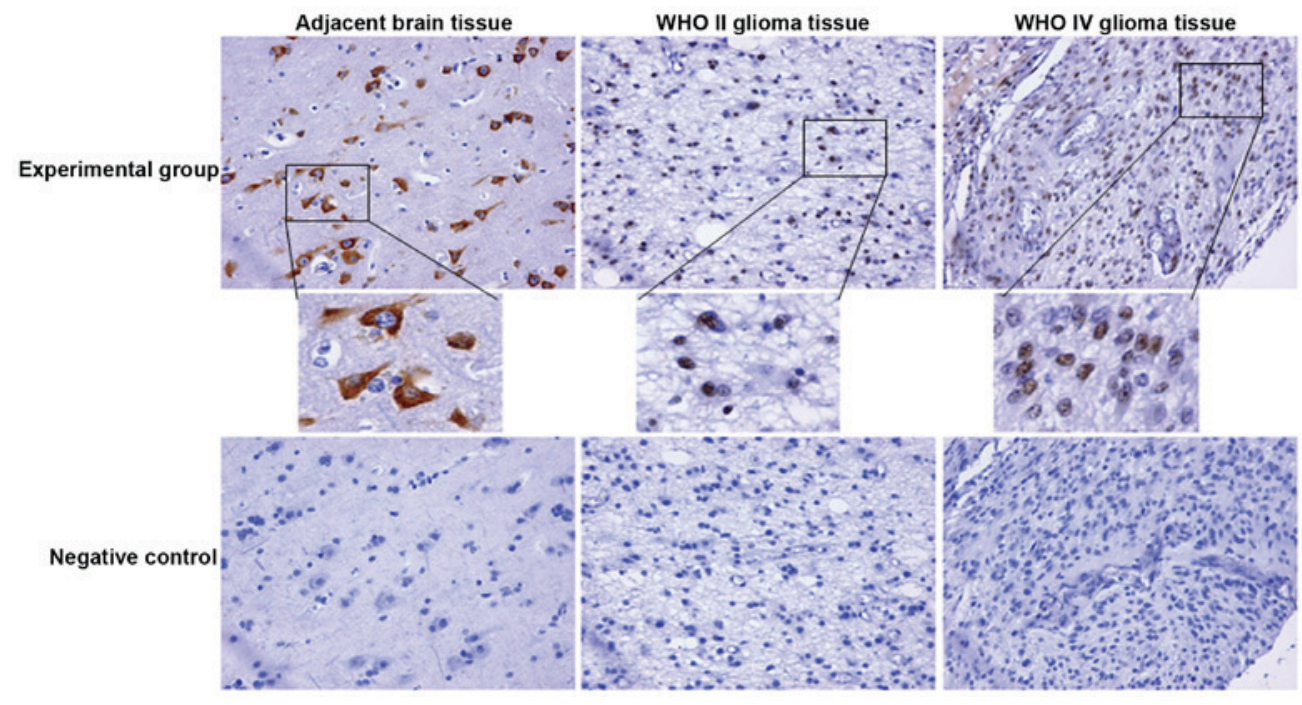

Figure 4. The protein expression and location of IRX1 in glioma tissues, detected by tissue microarray analysis.

can be used as controls for western blot test, the comparative analysis was meaningless. Therefore, western blot was not used to confirm the PCR results in the cell lines.

IRX1 is shown to play different roles in different cancer conditions (5). In hepatocellular carcinoma condition, Yang et al. identified IRX1 as a tumor oncogene in hepatocellular cancer, within which the IRX1 expression was increased and were related to the differentiation level of the cancer (9). IRX1 behaved as a prometastatic gene in osteosarcoma, the increased expression of which was associated with the cancer metastasis, via induction of CXCL14/NF- $\kappa \mathrm{B}$ signaling pathway (10). IRX1 was found down regulated in gastric cancer, and was labeled as tumor suppressor gene, the mechanism of which was found associated with the target genes FGF7, HIST2H2BE, and BDKRB2-dependent downstream gene PAK1 $(11,12)$. The tumor suppressor activity of IRX1 gene was also acknowledged in head and neck squamous cell carcinoma and pancreatic cancer $(13,14)$. In addition, IRX1 is known to induce transcription of HOXB4 gene, a downstream target gene of WNT, c-KIT and TPO pathways, and inhibit the function of MLL-AF4 and thus regulate acute lymphoblastic leukemia (15). MAPK pathway, in which PAK1 is a key protein,
$\mathrm{NF}-\kappa \mathrm{B}$ pathway and WNT pathway have demonstrated to influence glioma (16). With IRX1 shown to influence above pathways, it can be speculated that IRX1 might be involved in the development and progression of glioma through affecting some key molecules in these signaling pathways.

As per our study results, demonstrating an increased expression of IRX1 in glioma condition and its direct correlation with glioma grade, IRX1 can be labeled as tumor oncogene in glioma. The underlying molecular mechanism associated with the increased IRX1 expression in glioma cells and clinical glioma tissues is yet to be established. It has been reported that the global hypomethylation is a common mechanism in primary human glioblastomas (17). In support of the statement, several studies demonstrate the hypomethylation of various genes in glioma condition (18-22). The hypomethylation of IRX1 gene has been observed in metastatic osteosarcoma and gastric cancer $(10,11)$. Correspondingly, IRX1 hypomethylation could be a possible underlying molecular mechanism of the observed increase in its expression in glioma, which needs further assessments. In further mechanistic studies, we will focus on investigating how the increased expression of the protein is associated with the glioma progression. 
The occurrence of glioma is influenced by multi-factors, multi-stages and multi-genes (23). In-depth study of the tumor gene expression profile is of great significance in tumor diagnosis, treatment, and monitoring the recurrence and prognosis of glioma. Increased expression of IRX1, and its relocation to the nucleus in higher grade glioma, suggests of a direct influence of IRX1 in the proliferation, metastasis and development of glioma, though the underlying mechanism is not clear yet. If hypomethylation triggers the increase in IRX1 expression and if so, at what stage of glioma, needs to be determined yet. Further, the molecules/genes among the MAPK, NF- $\kappa \mathrm{B}$, WNT, and other activated pathways through which the IRX1 affects glioma progression remains to be studied further.

\section{Acknowledgements}

National Natural Scientifc Foundation of China for Yanyang Tu (No. 81572983), Social Development of Technology Research Projects in Shaanxi Province for Pengxing Zhang (No. 2015SF027), Social Development of Technology Research Projects in Shaanxi Province for Hui Liu (No. 2016SF191), and Beijing Key Laboratory of Brain Major Diseases Open Project for Yanyang Tu (2015). Foundation of science innovation and development in Tangdu Hospital, Fourth Military Medical University for Nan Liu (No. 2016JCYJ013).

\section{References}

1. Minniti G, De Sanctis V, Muni R, Filippone F, Bozzao A Valeriani M, Osti MF, De Paula U, Lanzetta G, Tombolini V and Maurizi Enrici R: Radiotherapy plus concomitant and adjuvant temozolomide for glioblastoma in elderly patients. J Neurooncol 88: 97-103, 2008.

2. Nakada M, Nakada S, Demuth T, Tran NL, Hoelzinger DB and Berens ME: Molecular targets of glioma invasion. Cell Mol Life Sci 64: 458-478, 2007.

3. Bürglin TR: Analysis of TALE superclass homeobox genes (MEIS, PBC, KNOX, Iroquois, TGIF) reveals a novel domain conserved between plants and animals. Nucleic Acids Res 25: 4173-4180, 1997.

4. Doi T, Lukošiūtė A, Ruttenstock E, Dingemann J and Puri P: Expression of Iroquois genes is up-regulated during early lung development in the nitrofen-induced pulmonary hypoplasia. J Pediatr Surg 46: 62-66, 2011.

5. Zhang PX, Yang HW, Wang X, Wang L, Cheng YD,Zhang YS, et al: The genomic organization and function of IRX1 in tumorigenesis and development. Cancer Transl Med 3: 29-33, 2017.

6. Allen M, Bjerke M, Edlund H, Nelander S and Westermark B: Origin of the U87MG glioma cell line: Good news and bad news. Sci Transl Med 8: 354re3, 2106.
7. Torsvik A, Stieber D, Enger PØ, Golebiewska A, Molven A, Svendsen A, Westermark B, Niclou SP, Olsen TK, Chekenya Enger $\mathrm{M}$ and Bjerkvig R: U-251 revisited: genetic drift and phenotypic consequences of long-term cultures of glioblastoma cells. Cancer Med 3: 812-824, 2014.

8. Tu Y, Gao X, Li G, Fu H, Cui D, Liu H, Jin W and Zhang Y: MicroRNA-218 inhibits glioma invasion, migration, proliferation, and cancer stem-like cell self-renewal by targeting the polycomb group gene Bmil. Cancer Res 73: 6046-6055, 2013.

9. Yang HJ, Yu S, Wang J, Chen C, Gu Y and Bao XY: Expression and clinical significance of Iroquois homebox gene IRX1 in human hepatocellular carcinoma cells. Chin J Biologicals 25: 1354-1357, 2010 (In Chinese).

10. Lu J, Song G, Tang Q, Zou C, Han F, Zhao Z, Yin J, Xu H, Xie X et al: IRX1 hypomethylation promotes osteosarcoma metastasis via induction of CXCL14/NF- $\kappa$ B signaling. J Clin Invest 25: 1839-1856, 2015.

11. Guo X, Liu W, Pan Y, Ni P, Ji J, Guo L, Zhang J, Wu J, Jiang J, Chen X, et al: Homeobox gene IRX1 is a tumor suppressor gene in gastric carcinoma. Oncogene 29: 3908-3920, 2010.

12. Jiang J, Liu W, Guo X, Zhang R, Zhi Q, Ji J, et al: IRX1 influences peritoneal spreading and metastasis via inhibiting BDKRB2-dependent neovascularization on gastric cancer. Oncogene 30: 4498-4508, 2011.

13. Bennett KL, Karpenko M,Lin MT, Claus R, Arab K, Dyckhoff G, Plinkert P, Herpel E, Smiraglia D and Plass C: Frequently methylated tumor suppressor genes in head and neck squamous cell carcinoma. Cancer Res 68: 4494-4499, 2008.

14. Wei W, Xu L, Wang F, He SS, Yang LJ, Guo CY and Wang XP: Expression and methylation of Iroquois homeobox protein 1 in pancreatic cancer. Chin J Pancreat 11: 309-311, 2011.

15. Kühn A, Löscher D and Marschalek R: The IRX1/HOXA connection: insights into a novel $\mathrm{t}(4 ; 11)$-specific cancer mechanism. Oncotarget 7: 35341-35352, 2016.

16. Cheng YD, Tu YY and Liang P: Promoter Methylated Tumor Suppressor Genes in Glioma. Cancer Transl Med 1: 123-130, 2015.

17. Cadieux B, Ching TT, VandenBerg SR and Costello JF: Genome-wide hypomethylation in human glioblastomas associated with specific copy number alteration, methylenetetrahydrofolate reductase allele status, and increased proliferation. Cancer Res 66: 8469-76, 2006.

18. Ting AH, McGarvey KM and Baylin SB: The cancer epigenomecomponents and functional correlates. Genes Dev 20: 3215-3231, 2006.

19. Jones PA and Baylin SB: The epigenomics of cancer. Cell 128: 683-692, 2007.

20. Frigola J, Solé X, Paz MF, Moreno V, Esteller M, Capellà G and Peinado MA: Differential DNA hypermethylation and hypomethylation signatures in colorectal cancer. Hum Mol Genet 14: 319-326, 2005 .

21. Ehrlich M, Jiang G, Fiala E, Dome JS, Yu MC, Long TI, Youn B, Sohn OS, Widschwendter M, Tomlinson GE, et al: Hypomethylation and hypermethylation of DNA in Wilms tumors. Oncogene 21: 6694-6702, 2002.

22. Herman JG and Baylin SB: Gene silencing in cancer in association with promoter hypermethylation. N Engl J Med 349: 2042-2054, 2003.

23. Liu N and Tu YY: Systematic Review of MicroRNAs and its Therapeutic Potential in Glioma. Cancer Transl Med 1: 50-66, 2015. 